\title{
Molecular Dissection of the Semaphorin 4D Receptor Plexin- B1-Stimulated R-Ras GTPase-Activating Protein Activity and Neurite Remodeling in Hippocampal Neurons
}

\author{
Izumi Oinuma, Hironori Katoh, and Manabu Negishi \\ Laboratory of Molecular Neurobiology, Graduate School of Biostudies, Kyoto University, Kyoto 606-8502, Japan
}

\begin{abstract}
Plexins serve as receptors for repulsive axonal guidance molecules semaphorins. The cytoplasmic domain of the semaphorin 4D (Sema4D) receptor, Plexin-B1 has two separated Ras GTPase-activating protein (GAP)-homologous domains, C1 and C2. Recently, we reported that the Rho family small GTPase Rnd1 associates with Plexin-B1, and the Plexin-B1-Rnd1 complex stimulates GTPase activity of R-Ras, inducing growth cone collapse in hippocampal neurons in response to Sema4D. However, the molecular mechanisms by which Plexin-B1 exhibits the GAP activity remain unclear. In this report, critical roles of Rnd1 and Sema4D in Plexin-B1-stimulated R-Ras GAP activity and neurite remodeling were examined. The $\mathrm{N}$-terminal region of the cytoplasmic domain of Plexin- $\mathrm{B} 1$ containing the $\mathrm{C} 1$ domain interacts with the $\mathrm{C}$-terminal region containing the $\mathrm{C} 2$ domain, and Rnd1 disrupts this interaction. On the other hand, Sema4D induces clustering of Rnd1-bound Plexin-B1, in parallel with inactivation of R-Ras in cells. Antibody clustering of the recombinant cytoplasmic domain of Plexin-B1 in the presence of Rnd1 triggers the R-Ras GAP activity. Deletion of the extracellular domain of Plexin-B1 causes ligand-independent clustering of the receptor, rendering the receptor constitutively active in the presence of Rnd1, and induces contraction of COS-7 cells and inhibition of neurite outgrowth in hippocampal neurons. These results indicate that Rnd1 opens the two R-Ras GAP domains of Plexin-B1, and Sema4D-induced receptor clustering stimulates R-Ras GAP activity and neurite remodeling in hippocampal neurons.
\end{abstract}

Key words: plexin; semaphorin; neurite outgrowth; Rho; R-Ras; GAP

\section{Introduction}

During development of the nervous system, axons are guided to their proper targets by sensing a variety of extracellular cues in the local environment. Neuronal growth cones, located at the tip of the growing axon, are highly motile structures and respond to attractive and repulsive cues by selectively altering the stability of the actin cytoskeleton.

Semaphorins are a large family of secreted or membranebound proteins (Nakamura et al., 2000), which have been shown to regulate axonal pathfinding during the development of the nervous system (Kolodkin et al., 1993; Luo et al., 1993; Raper, 2000). The function of semaphorins is mediated by Plexins, which are classified into four subfamilies: Plexin-A1-4, PlexinB1-3, Plexin-C1, and Plexin-D1 (Tamagnone et al., 1999). Plexin-B1 has been identified as a receptor for semaphorin 4D (Sema4D, also known as CD100) (Tamagnone et al., 1999). Recent studies indicate that Plexin-B1 forms a complex with PDZ-Rho guanine nucleotide exchange factor (GEF)/leukemia-associated RhoGEF (LARG) via its C-terminal PDZ (postsynaptic density-95/

Received Aug. 9, 2004; revised 0ct. 21, 2004; accepted Nov. 12, 2004.

This work was supported by grants-in-aid for scientific research from the Ministry of Education, Science, Sports, and Culture of Japan.

Correspondence should be addressed to Manabu Negishi, Laboratory of Molecular Neurobiology, Graduate School of Biostudies, Kyoto University, Sakyo-ku, Kyoto 606-8502, Japan. E-mail: mnegishi@pharm.kyoto-u.ac.jp. DOI:10.1523/JNEUROSCI.3257-04.2004

Copyright $\odot 2004$ Society for Neuroscience $\quad 0270-6474 / 04 / 2411473-08 \$ 15.00 / 0$
Discs large/zona occludens-1) domain-binding motif (Aurandt et al., 2002; Hirotani et al., 2002; Perrot et al., 2002; Swiercz et al., 2002). Sema4D induces growth cone collapse in hippocampal neurons (Swiercz et al., 2002; Oinuma et al., 2004) and neurite retraction in PC12 cells (Perrot et al., 2002; Oinuma et al., 2004), and these events are, in part, mediated by RhoGEF-mediated RhoA activation.

Rho family small GTPases are signal transduction molecules that remodel the actin cytoskeleton and play fundamental roles in numerous cellular processes initiated by extracellular stimuli, including axonal guidance (Etienne-Manneville and Hall, 2002; Negishi and Katoh, 2002). The small GTPase Rnd1, known as a constitutively active GTPase (Nobes et al., 1998), and the GTPbound form of Rac are known to interact directly with the cytoplasmic domain of Plexin-B1 (Vikis et al., 2000; Drissens et al., 2001). Of them, Rnd1 has been shown to form a constitutive, ligand-independent interaction with Plexin-B1, to stimulate Sema4D-mediated repulsive responses via the promotion of the interaction between Plexin-B1 and PDZ-RhoGEF (Oinuma et al., 2003), and also to cause the increased cell surface expression of Plexin-B1 (Swiercz et al., 2004).

Activation of Plexin-B1 is also known to hinder integrinbased cell substrate adhesion, independent of RhoA activation (Barberis et al., 2004). We recently reported that Plexin-B1 encodes a GTPase-activating protein (GAP) toward R-Ras in the cytoplasmic domain, and the Plexin-B1-Rnd1 complex mediates Sema4D-induced repulsive signaling by stimulating the intrinsic 
GTPase activity of R-Ras in hippocampal neurons (Oinuma et al., 2004). R-Ras has been shown to promote cell adhesion and neurite outgrowth by activating integrins (Zhang et al., 1996; Ivins et al., 2000; Kinbara et al., 2003). Both Rnd1 binding to the receptor and Sema4D stimulation are indispensable for this GAP activity (Oinuma et al., 2004), but critical roles of Rnd1 and Sema4D in the function of Plexin-B1 still remain unclear. Here we show that Rnd1 opens the two R-Ras GAP domains of Plexin-B1, and Sema4D-induced receptor clustering stimulates R-Ras GAP activity and neurite remodeling in hippocampal neurons.

\section{Materials and Methods}

Expression plasmids and antibodies. Hemagglutinin (HA)- and green fluorescent protein (GFP)-tagged Rnd1, HA-tagged Rnd1-A45, HA-tagged human R-Ras, the glutathione $S$-transferase (GST)-tagged Ras-binding domain of c-Raf-1 (amino acids 53-130), Myc-tagged Plexin-B1, PlexinB1-GGA (L1849G, V1850G, P1851A), and Plexin-B1-RA (R1677A, R1678A, R1984A) have been described previously (Oinuma et al., 2004). HA-tagged Plexin-B1 was kindly provided by Dr. S. Inagaki (Osaka University, Osaka, Japan). PCR-amplified fragments were ligated into a vector containing an Ig $\kappa$-chain leader secretion signal sequence at the $\mathrm{N}$ terminus to express Plexin-B1 $\Delta$ sem (deletion of amino acids 127-516), $\Delta \operatorname{Ig}$ (deletion of amino acids $1160-1376$ ), and $\Delta$ ect (deletion of amino acids 1-1306). The N-terminal Myc-tagged cytoplasmic domain of Plexin-B1 (amino acids 1511-2136) was subcloned into pcDNA3 (Invitrogen, San Diego, CA) and pGEX-6P-1 (Amersham Biosciences, Arlington Heights, IL). GST fusion fragments of the cytoplasmic domain of Plexin-B1, Plexin-B1-N-Cyt (amino acids 1511-1915) and Plexin-B1-CCyt (amino acids 1916-2136), were generated by PCR amplification and subcloned into pGEX-4T-2. A soluble form of Sema4D fused to human $\mathrm{IgG}_{1}-\mathrm{Fc}$ was a generous gift from Dr. H. Kikutani (Osaka University). We used the following antibodies: mouse monoclonal antibodies against Myc (Upstate Biotechnology, Lake Placid, NY) and against GFP (Santa Cruz Biotechnology, Santa Cruz, CA); a rabbit polyclonal anti-Myc antibody (MBL, Nagoya, Japan); a rat monoclonal antibody against HA (Roche Molecular Biochemicals, Indianapolis, IN); secondary antibodies conjugated to horseradish peroxidase (Dako, Carpinteria, CA); and secondary antibodies conjugated to Alexa 594 (Molecular Probes, Eugene, OR).

Immunoblotting. Proteins were separated by 7 or $12.5 \%$ SDS-PAGE and were electrophoretically transferred onto a polyvinylidene difluoride membrane (Millipore, Bedford, MA). The membrane was blocked with $3 \%$ low-fat milk in Tris-buffered saline and then incubated with primary antibodies. The primary antibodies were detected by using horseradish peroxidase-conjugated secondary antibodies and an ECL detection kit (Amersham Biosciences).

Plexin-B1 receptor monomers and oligomers were separated by SDSPAGE in a $4-7 \%$ acrylamide gradient gel and analyzed under both reducing (with $\beta$-mercaptoethanol) and nonreducing (without $\beta$-mercaptoethanol) conditions to detect disulfide-linked oligomeric receptors as described elsewhere (Sorokin et al., 1994).

Cell culture and transfection. COS-7 cells were cultured in DMEM containing $10 \%$ fetal bovine serum, $4 \mathrm{~mm}$ glutamine, $100 \mathrm{U} / \mathrm{ml}$ penicillin, and $0.2 \mathrm{mg} / \mathrm{ml}$ streptomycin under humidified conditions in $95 \%$ air and $5 \% \mathrm{CO}_{2}$ at $37^{\circ} \mathrm{C}$. Transient transfections were performed with Lipofectamine 2000 (Invitrogen) according to the manufacturer's instructions. A soluble form of Sema4D was expressed as a fusion protein with the $\mathrm{Fc}$ fragment of human $\operatorname{IgG}_{1}$. Stimulation with Sema4D was performed by incubation of the cells with Sema4D-Fc-containing medium at $37^{\circ} \mathrm{C}$.

For COS-7 cell contraction assay, the size of transfected cells was determined from digital images acquired at $20 \times$ magnification by using a Leica (Nussloch, Germany) DC350F digital camera system equipped with a Nikon (Tokyo, Japan) Eclipse E800 microscope and Image-Pro Plus image analysis software (Media Cybernetics, Silver Spring, MD). GFP- and Myc-staining double-positive cells with an area of $<350 \mu \mathrm{m}^{2}$ were determined as contracted cells.

For the neurite outgrowth analysis, embryonic day 19 (E19) rat hippocampal neurons were isolated as described previously (Ishikawa et al.,
2003) and plated on laminin-coated coverslips. Transfections were performed after $2 \mathrm{~d}$ in culture with Lipofectamine 2000. One day later, the length of the longest neurite of the transfected cells was determined from digital images acquired at $40 \times$ magnification by using a Leica DC350F digital camera system equipped with Nikon Eclipse E800 microscope and Image-Pro Plus image analysis software (Media Cybernetics).

Pull-down assay and immunoprecipitation. All recombinant GST fusion proteins were purified from Escherichia coli as described (Katoh et al., 2002; Oinuma et al., 2003). Protein concentration was determined by comparing with bovine serum albumin standards after SDS-PAGE and by staining with Coomassie brilliant blue.

For pull-down assays with GST-Plexin-B1-N-Cyt and -C-Cyt, COS-7 cells $\left(7 \times 10^{5}\right.$ cells $)$ were rinsed once with PBS and lysed with ice-cold cell lysis buffer [20 mм Tris- $\mathrm{HCl}, \mathrm{pH}$ 7.5, 2 mм $\mathrm{MgCl}_{2}, 1 \% \mathrm{NP}-40,1 \mathrm{~mm}$ phenylmethylsulfonyl fluoride (PMSF), $1 \mathrm{~mm}$ dithiothreitol (DTT), 10 $\mu \mathrm{g} / \mathrm{ml}$ aprotinin, and $10 \mu \mathrm{g} / \mathrm{ml}$ leupeptin]. Cell lysates were then centrifuged for $10 \mathrm{~min}$ at $18,000 \times g$ at $4^{\circ} \mathrm{C}$. The supernatants were incubated for $10 \mathrm{~min}$ at $4^{\circ} \mathrm{C}$ with $10 \mu \mathrm{g}$ of GST fusion proteins and subsequently incubated with glutathione-Sepharose beads for $1 \mathrm{hr}$ at $4^{\circ} \mathrm{C}$. After the beads were washed twice with the ice-cold cell lysis buffer, the bound proteins were eluted in Laemmli sample buffer and analyzed by SDSPAGE and immunoblotting with antibody.

For immunoprecipitation assays of full-length Plexin-B1, COS-7 cells $\left(7 \times 10^{5}\right.$ cells) were lysed with ice-cold cell lysis buffer $(10 \mathrm{~mm}$ Tris- $\mathrm{HCl}$, $\mathrm{pH} 7.5,5$ mм $\mathrm{MgCl}_{2}, 2$ mм EDTA, 1\% NP-40, 1 mм PMSF, $10 \mu \mathrm{g} / \mathrm{ml}$ aprotinin, and $10 \mu \mathrm{g} / \mathrm{ml}$ leupeptin). After centrifugation, the supernatants were incubated with anti-Myc polyclonal antibody for $1 \mathrm{hr}$ and then with protein A-Sepharose (Amersham Biosciences) for $1 \mathrm{hr}$. The beads were washed once with lysis buffer, and bound proteins were analyzed by SDS-PAGE and immunoblotting.

Measurement of R-Ras activity. Measurement of GTPase activity in vitro was performed as described previously (Ohba et al., 2000; Oinuma et al., 2004). The purified recombinant Myc-tagged cytoplasmic domain of Plexin-B1 $(0.5 \mu \mathrm{g})$ was clustered at room temperature by mouse monoclonal anti-Myc antibody, followed by incubation with an antibody against mouse Igs. After the clustering reaction, the complex was incubated with recombinant Rnd $1(1 \mu \mathrm{g})$ for $30 \mathrm{~min}$, and then $20 \mathrm{ng}$ of $\mathrm{R}$-Ras preloaded with $\left[\gamma^{-32} \mathrm{P}\right] \mathrm{GTP}$ was added and used for the nitrocellulose filtration assay.

Measurement of R-Ras activity in cells was performed as described previously. COS-7 cells $\left(7 \times 10^{5}\right.$ cells) were maintained in DMEM containing $5 \%$ fetal bovine serum after transfection. Sixteen hours after transfection, cells were lysed in cell lysis buffer $(25 \mathrm{~mm}$ HEPES- $\mathrm{NaOH}$, pH 7.5, 150 mм NaCl, 1\% NP-40, 0.25\% Na-deoxycholate, 0.1\% SDS, $10 \%$ glycerol, $10 \mathrm{~mm} \mathrm{MgCl}_{2}$, 1 mм EDTA, 1 mm DTT, $10 \mu \mathrm{g} / \mathrm{ml}$ aprotinin, and $10 \mu \mathrm{g} / \mathrm{ml}$ leupeptin) containing $75 \mu \mathrm{g}$ of GST-fused Rasbinding domain of c-Raf-1 (GST-RBD).

Detection of receptor clustering. Sema4D-induced clustering of Plexin-B1 was examined by immunoprecipitation assay using full-length receptors tagged with HA or Myc. COS-7 cells were cotransfected with HA- and Myc-tagged receptors, and at $36 \mathrm{hr}$ after transfection, clustering of the receptors was analyzed by immunoprecipitation, followed by Western blotting.

To determine spontaneous clustering states of deletion mutants of Plexin-B1, COS7 cells $\left(7 \times 10^{5}\right.$ cells) were collected $48 \mathrm{hr}$ after transfection, rinsed once with PBS, and resuspended in ice-cold lysis buffer (10 mu Tris- $\mathrm{HCl}, \mathrm{pH}$ 7.4, 2 mм EDTA, 5\% glycerol, $10 \mu \mathrm{g} / \mathrm{ml}$ aprotinin, and $10 \mu \mathrm{g} / \mathrm{ml}$ leupeptin). Cell lysates were frozen in liquid nitrogen, thawed at $4^{\circ} \mathrm{C}$, and then centrifuged for $10 \mathrm{~min}$ at $18,000 \times g$ at $4^{\circ} \mathrm{C}$ to remove the supernatants. The same process was repeated twice to remove the cytosolic fraction, and then the pellets were analyzed by SDS-PAGE and immunoblotting both under reducing and nonreducing conditions.

\section{Results}

Rnd1 disrupts the interaction between the $\mathrm{N}$ - and C-terminal regions within the cytoplasmic domain of Plexin-B1

The Rnd1-binding region in Plexin-B1 splits the R-Ras GAP domain into $\mathrm{C} 1$ and $\mathrm{C} 2$ domains, which contain primary and secondary arginine motifs, respectively, essential for the catalytic 
A

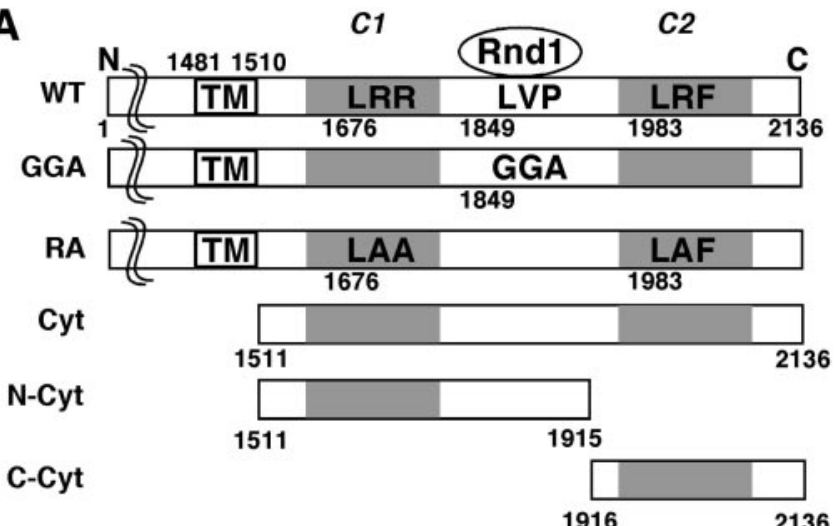

B

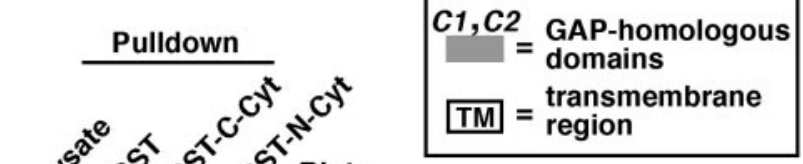

C

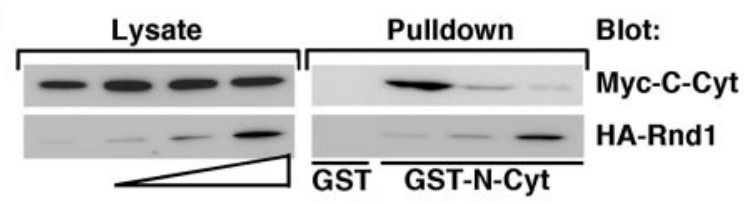

D

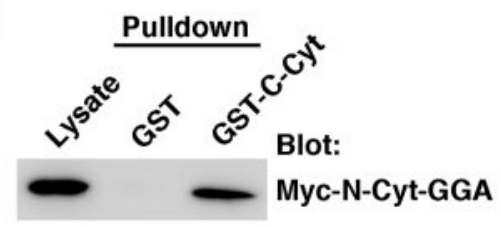

E

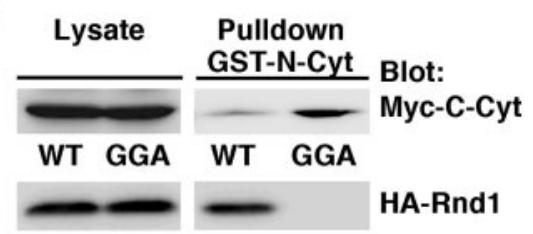

$\mathbf{F}$

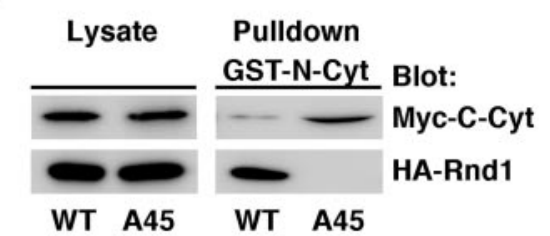

G

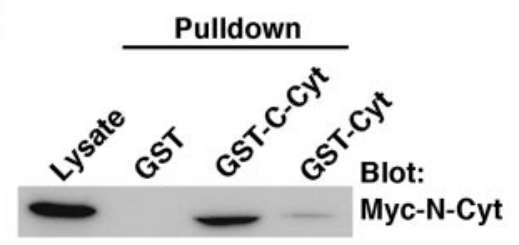

Figure 1. Rnd1 disrupts the interaction between the $\mathrm{N}$ - and C-terminal regions within the cytoplasmic domain of Plexin-B1.A, Schematic representation of the Plexin-B1 constructs used in this study. The Rnd1-binding region and the R-Ras GAP domain are indicated. Letters indicate specific amino acid residues within domains ( $A$, Ala; F, Phe; $G, G l y ; L$, Leu; P, Pro; R, Arg; V, Val), and numbers indicate amino acid positions within the sequence. $B$, Lysates from COS-7 cells expressing the Myc-tagged $\mathrm{N}$-terminal portion of the cytoplasmic domain of Plexin-B1 activity of R-Ras GAP (Fig. 1A). To understand a role of Rnd1 binding in conformation of the cytoplasmic domain of Plexin$\mathrm{B} 1$, we examined the interaction between the $\mathrm{N}$-terminal region (amino acids 1511-1915; N-Cyt) and C-terminal region (amino acids 1916-2136; C-Cyt) of the cytoplasmic domain of Plexin$\mathrm{B} 1$, each of which contains $\mathrm{C} 1$ and $\mathrm{C} 2$ domains, respectively (Fig. $1 A)$. Myc-tagged N-Cyt was expressed in COS-7 cells, and a pulldown assay was performed with purified GST-fused C-Cyt. As shown in Figure $1 B$, N-Cyt interacted with C-Cyt but not with $\mathrm{N}$-Cyt itself, suggesting that the $\mathrm{N}$-terminal region specifically interacts with the C-terminal region within the cytoplasmic domain of Plexin-B1. To examine the effect of Rnd 1 binding on this interaction, Myc-tagged C-Cyt and increasing amounts of HAtagged wild-type Rnd1 were expressed in COS-7 cells, and a pulldown assay was performed by using GST-N-Cyt. Rnd 1 decreased the interaction of $\mathrm{N}$ - and $\mathrm{C}$-Cyt in a concentration-dependent manner (Fig. 1C). C-Cyt also associated with N-Cyt-GGA, which has no ability to interact with Rnd1 (Fig. $1 D$ ), and Rnd1 could not disrupt this interaction (Fig. $1 E$ ). In addition, cotransfection of Rnd $1^{\mathrm{A} 45}$, a mutant of Rnd 1 that contains a T45A substitution in the effector domain and does not interact with Plexin-B1, had no effect on the interaction between N- and C-Cyt (Fig. 1 F). These results indicate that Rnd 1 disrupts the interaction between the $\mathrm{C} 1$-containing $\mathrm{N}$-terminal region and the $\mathrm{C} 2$-containing C-terminal region within the cytoplasmic domain of Plexin-B1. We also found that, compared with GST-fused C-Cyt, the GSTfused full length of the Plexin-B1 cytoplasmic domain exhibited a weak interaction with $\mathrm{N}$-Cyt (Fig. $1 G$ ). This result also supports the idea that the cytoplasmic domain of Plexin-B1 takes the intramolecularly tethered form.

\section{Sema4D induces clustering of Plexin-B1 in parallel with inactivation of R-Ras}

We next examined the role of Sema4D in Plexin-B1 activation. To evaluate whether Plexin-B1 is clustered by Sema4D, we introduced full-length Plexin-B1 carrying two different epitopes, HA and $\mathrm{Myc}$, and performed an immunoprecipitation assay. COS-7 cells were transfected with an HA-tagged Plexin-B1 receptor and a Myc-tagged one together with GFP-tagged Rnd1, and the cell lysates were immunoprecipitated with anti-Myc antibody to analyze receptor-receptor interaction. After stimulation with Sema4D, the interaction between $\mathrm{HA}^{-}$and Myc-tagged Plexin-B1 increased, and the level reached the maximum within 5 min and then decreased thereafter (Fig. 2A). We next examined the time course of the R-Ras GAP activity of Plexin-B1 by a pulldown assay with the GST-fused Ras-binding domain of c-Raf-1, which can selectively isolate active R-Ras (van Triest et al., 2001; Cole et al., 2003). After stimulation with Sema4D, the level of GTP-bound R-Ras in the cells decreased and reached the minimum within $5 \mathrm{~min}$, the basal level being regained afterward (Fig.

\footnotetext{
$\leftarrow$

(Myc-N-Cyt) were used in a pull-down assay with the GST-fused C-terminal portion (GST-C-Cyt). Bound proteins and total cell lysate (Lysate) were analyzed by immunoblotting with anti-Myc antibody. C, Myc-tagged C-Cyt and increasing amounts of HA-tagged Rnd1 were expressed in COS-7 cells. Lysates were used in a pull-down assay with GST-N-Cyt. D, Lysates from COS-7 cells expressing Myc-N-Cyt-GGA were used in a pull-down assay with GST-C-Cyt. E, Lysates from COS-7 cells expressing Myc-C-Cyt and HA-tagged Rnd1 were used in a pull-down assay with wild-type (WT) or the GGA mutant of GST-N-Cyt. F, Lysates from COS-7 cells expressing Myc-CCyt and wild-type or the A45 mutant of HA-tagged Rnd1 were used in a pull-down assay with GST-N-Cyt. G, Lysates from COS-7 cells expressing Myc-N-Cyt were used in a pull-down assay with GST-Cyt. The results shown are representative of three independent experiments that yielded similar results.
} 
A

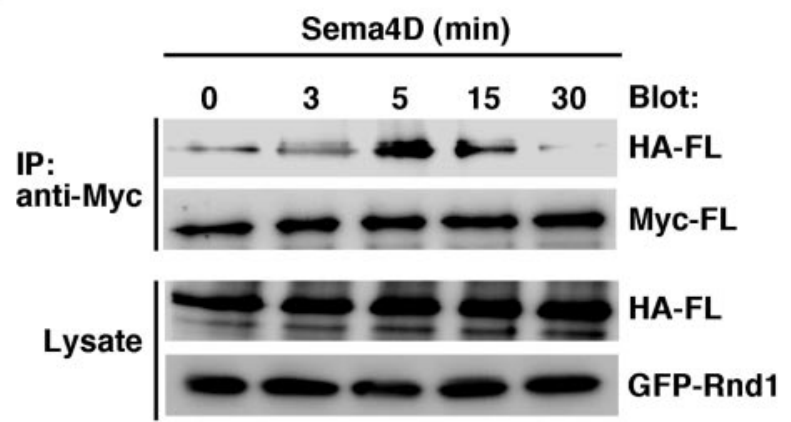

B

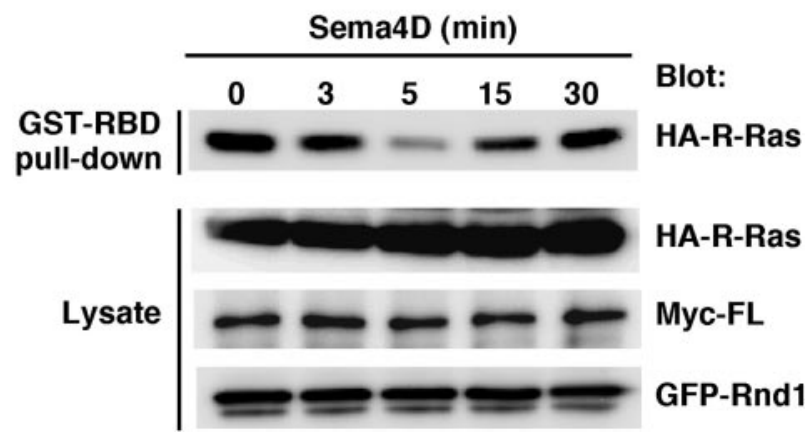

Figure 2. Sema $4 \mathrm{D}$ induces clustering of Plexin- $\mathrm{B} 1$ in parallel with inactivation of R-Ras. $A$, COS-7 cells transfected with Myc- and HA-tagged full-length Plexin-B1 and GFP-tagged Rnd1 were stimulated with Sema4D for the indicated times. The cell lysates were immunoprecipitated (IP) with anti-Myc antibody. B, COS-7 cells transfected with Myc-Plexin-B1, GFP-Rnd1, and HA-R-Ras were stimulated with Sema4D for the indicated times. Then the cell lysates were incubated with GST-RBD, and bound R-Ras was detected with anti-HA antibody. The results shown are representative of five independent experiments that yielded similar results. FL, Full length.

$2 B$ ). These results indicate that Sema4D induces clustering of Plexin-B1 in parallel with inactivation of R-Ras.

Antibody clustering of the cytoplasmic domain of Plexin-B1 causes R-Ras GAP activity in the presence of Rnd1 in vitro To understand the functional importance of the clustering and Rnd1 binding for the GAP activity of Plexin-B1, the Myc-tagged recombinant cytoplasmic domain of Plexin-B1 prepared from $E$. coli was directly clustered by mouse monoclonal antibody against Myc and an antibody against mouse Igs, with or without Rnd1. Recombinant R-Ras preloaded with $\left[\gamma_{-}{ }^{32} \mathrm{P}\right] \mathrm{GTP}$ was incubated with this complex, and GTPase activity of R-Ras was determined. As shown in Figure 3, GTPase activity of R-Ras was not altered by clustering of Plexin-B1 alone. In contrast, Rnd1-bound Plexin-B1 blocked the basal GTPase activity of R-Ras, probably because of trapping of GTP-bound active R-Ras, and clustering of Rnd1-bound Plexin-B1 could dramatically stimulate hydrolysis of bound GTP of R-Ras. On the other hand, clustering of either Plexin-B1-GGA or Plexin-B1-RA had no effect in the presence of Rnd1. These results suggest that the clustering of the Rnd1-bound Plexin-B1 is necessary and sufficient for displaying R-Ras GAP activity of Plexin-B1.

Extracellular domain-deleted Plexin-B1 forms a disulfidelinked covalent oligomer, displaying constitutive activity of R-Ras GAP

Plexins have a sema domain and three Ig-like domains in the extracellular region (Fig. 4A). The sema domain of Plexin-A1 is

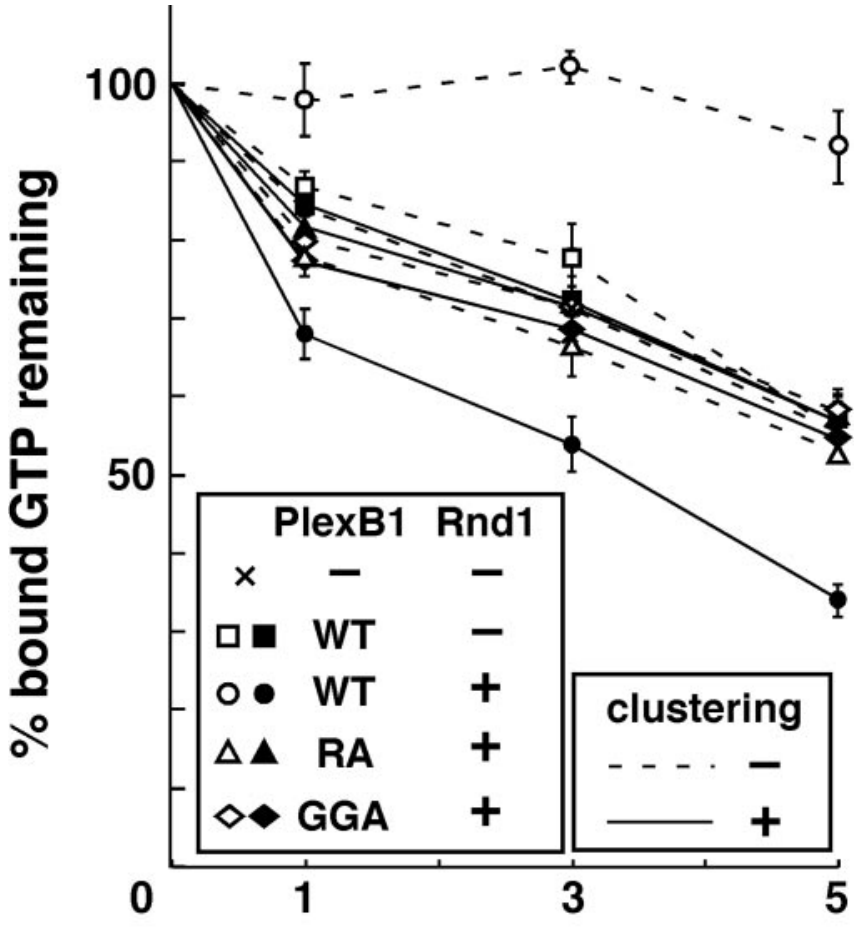

\section{Time after clustering (min)}

Figure 3. Antibody clustering of the cytoplasmic domain of Plexin-B1 stimulates the GTPase activity of R-Ras. Recombinant R-Ras preloaded with $\left[\gamma^{-32}\right.$ P]GTP was incubated with the recombinant cytoplasmic domain of Plexin-B1 with (filled symbols, solid lines) or without (empty symbols, dashed lines) antibody-based clustering. The remaining radioactivity bound to R-Ras on the indicated times was measured by a nitrocellulose filtration assay. WT, Wild type.

known to be an autoinhibitory domain, and sema domaindeleted Plexin-A1 acts as a constitutively activated receptor (Takahashi and Strittmatter, 2001). Deletion of the Ig-like domain is also known to cause clustering of receptors and constitutive activation in some receptors (Jing et al., 1992; Arevalo et al., 2000).

We then generated the deletion mutants of Plexin-B1, in which the sema domain (deletion of amino acids 127-516; Plexin-B1 $\Delta$ sem), the second Ig-like domain, the third Ig-like domain (deletion of amino acids 1160-1376; Plexin-B1 $\Delta \mathrm{Ig}$ ), or the entire extracellular region of Plexin-B1 (deletion of amino acids 1-1306; Plexin-B1 $\Delta$ ect) was deleted (Fig. 4A). These mutants were expressed in COS-7 cells and analyzed by SDS-PAGE and immunoblotting both under reducing and nonreducing conditions to determine the formation of spontaneous disulfide-linked oligomers (Fig. $4 B$ ). Among these mutants, only Plexin-B1 $\Delta$ ect formed disulfide-linked oligomers.

To determine whether these mutants display constitutive activity of R-Ras GAP, COS-7 cells were cotransfected with the mutants of Plexin-B1 and Rnd1, and then GTP-bound active $\mathrm{R}$-Ras in the cells was measured by a pull-down assay with GST-RBD (Fig. $4 C, D$ ). Plexin-B1 $\Delta$ ect, but neither $\Delta$ Ig nor $\Delta$ sem, decreased the GTP-R-Ras level in the cells in the absence of Sema4D, indicating that the $\Delta$ ect mutant is a constitutively active form in R-Ras GAP activity.

To evaluate the requirement of Rnd 1 in R-Ras GAP activity by Plexin-B1 $\Delta$ ect, COS-7 cells were transfected with Plexin-B1 $\Delta$ ect in the presence or absence of Rnd1. As shown in Figure 5, PlexinB1 1 ect decreased R-Ras activity in the presence of Rnd1 but not in the absence of Rnd1. We further examined R-Ras GAP activity

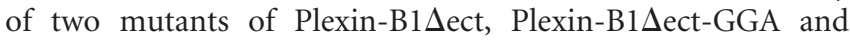


A

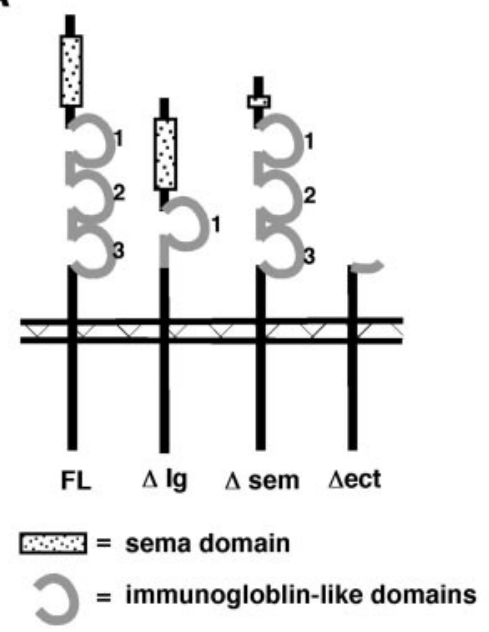

C

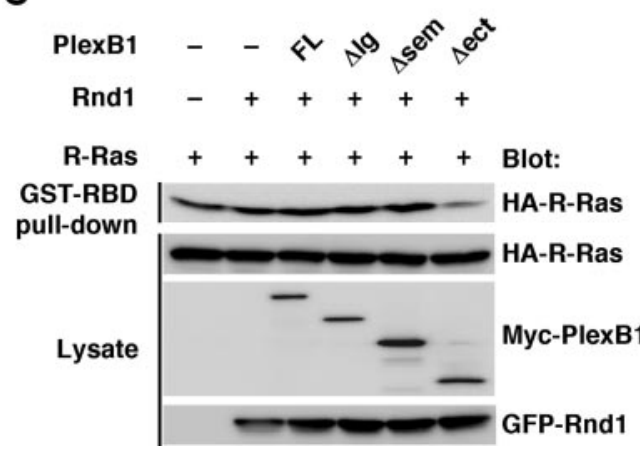

B

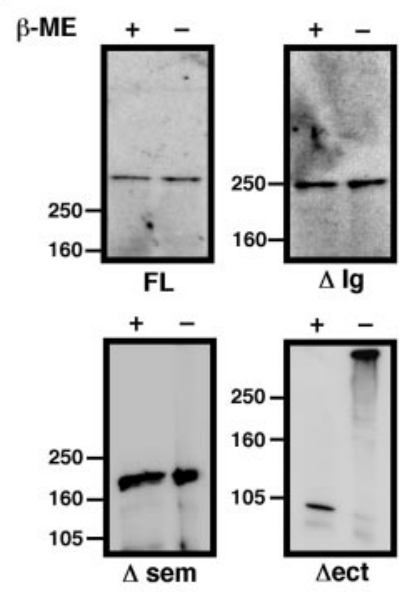

D

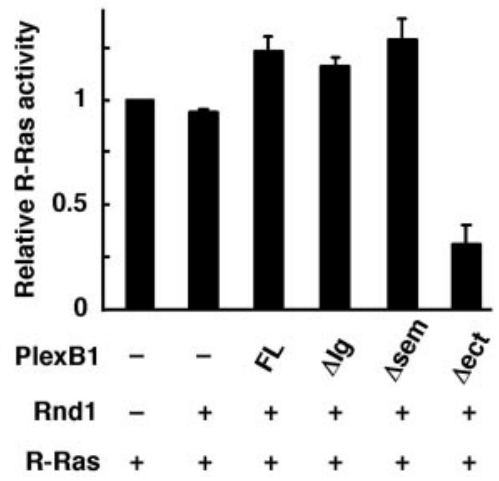

Figure 4. Deletion of the extracellular domain of Plexin-B1 induces ligand-independent clustering, leading to constitutive activation. $A$, Schematic representation of Plexin-B1 deletion constructs used in this study. $B$, The membrane fraction from COS-7 cells transfected with Plexin-B1 mutant receptors was analyzed by SDS-PAGE and immunoblotting both under reducing [with $\beta$-mercaptoethanol ( $\beta$-ME)] and nonreducing (without $\beta$-mercaptoethanol) conditions. C, Lysates from COS-7 cells expressing Myc-tagged Plexin-B1 mutants, GFP-Rnd1, and HA-R-Ras were incubated with GST-RBD, and bound R-Ras was detected with anti-HA antibody. D, Relative R-Ras activity was determined by the amount of R-Ras in cell lysates analyzed by NIH Image software. Results are the means \pm SEM of three independent experiments. FL, Full length.

Plexin-B1 $\Delta$ ect-RA; the former has no ability to interact with Rnd 1 , and the latter lacks primary and secondary arginine motifs essential for the catalytic activity of R-Ras GAP. Both mutants failed to decrease R-Ras activity regardless of the presence of Rnd1 (Fig. 5), although these mutants formed disulfide-linked oligomers (data not shown). These results indicate that Rnd1 binding is required for clustered Plexin-B1 $\Delta$ ect-induced GAP activity.

Plexin-B1Dect is constitutively active in contraction of COS-7 cells and neurite remodeling in hippocampal neurons

Semaphorins have been shown to induce contraction of the cell body of COS-7 cells expressing Plexins, and this morphological effect is widely used as a model system to study the repulsive signaling mediated by semaphorins (Takahashi et al., 1999; Rohm et al., 2000; Oinuma et al., 2003).

We then examined the Sema4D-independent contraction of COS-7 cells expressing the $\Delta \mathrm{Ig}, \Delta$ sem, or $\Delta$ ect mutant with or without Rnd1 (Fig. 6). Expression of Plexin-B1 $\Delta$ Ig or $\Delta$ sem failed to induce contraction of COS-7 cells regardless of the expression

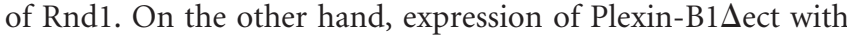
Rnd1 induced strong cell contraction, but this event was lost in the absence of Rnd1. In these experiments, immunostaining with
Plexin-B1 showed that expression levels between various constructs were similar (data not shown).

Sema4D induces growth cone collapse in primary hippocampal neurons from rat embryos (Swiercz et al., 2002; Oinuma et al., 2004), and growth cone collapse by semaphorins is also associated with reduced neurite outgrowth (Takahashi and Strittmatter, 2001). We recently revealed that Sema4D induces growth cone collapse via inactivation of R-Ras by the Plexin-B1Rnd1 complex in rat hippocampal neurons endogenously expressing Plexin-B1 and Rnd1 (Oinuma et al., 2004).

To confirm that the Plexin-B1 $\Delta$ ect mutant is constitutively active in neuronal cells as well as in COS-7 cells, we transfected E19 rat primary hippocampal neurons with Plexin-B1 $\Delta$ ect and examined morphological effects of the $\Delta$ ect mutant. Neurites of Plexin-B1 $\Delta$ ect-expressing hippocampal neurons were significantly shorter than those of full-length PlexinB1-expressing neurons (Fig. 7). This morphological effect was completely lost in the cells expressing Plexin-B1 $\Delta$ ect-RA, which lacks R-Ras GAP activity, or PlexinB1 1 ect-GGA, which has no ability to interact with Rnd1. In these experiments, immunostaining with Plexin-B1 showed that expression levels between various constructs were similar (data not shown). These results indicate that Plexin-B1 $\Delta$ ect is constitutively active in neurite remodeling in hippocampal neurons, and this activity requires the Rnd1-Plexin-B1 interaction and the R-Ras GAP domain of Plexin-B1.

\section{Discussion}

Plexin-B1 encodes R-RasGAP in the cytoplasmic domain, and R-RasGAP domain splits into $\mathrm{C} 1$ and $\mathrm{C} 2$ domains by the Rnd1binding domain (Fig. 1A). Rnd1 is a constitutively active Rho family member (Nobes et al., 1998) and shows a constitutive, ligand-independent interaction with Plexin-B1 in cells (Oinuma et al., 2003). The expression of R-RasGAP activity of Plexin-B1 and subsequent growth cone collapse require the Rnd1 binding to Plexin-B1 and Sema4D stimulation. Here we show that Rnd1 disrupts the interaction between $\mathrm{C} 1$ and $\mathrm{C} 2$ domains, and Sema4D induces clustering of the Plexin-B1-Rnd1 complex, displaying R-RasGAP activation and neurite remodeling. We propose that signaling of Plexin-B1 is initiated by two steps, Rnd1 binding and Sema4D-induced receptor clustering.

Biochemical and structural studies of catalytic domains of a variety of GAPs for Ras family GTPases reveal that catalytic domains contain two invariant arginine motifs, primary and secondary motifs, both of which are essential for the binding to Ras family GTPases and GAP activity (Scheffzek et al., 1998). In the case of the cytoplasmic domain of Plexin-B1, these primary and secondary arginine motifs are separately located in C1 and C2 domains. Here we revealed that the $\mathrm{C} 1$-containing $\mathrm{N}$-terminal region interacts with the $\mathrm{C} 2$-containing $\mathrm{C}$-terminal region of the 
A

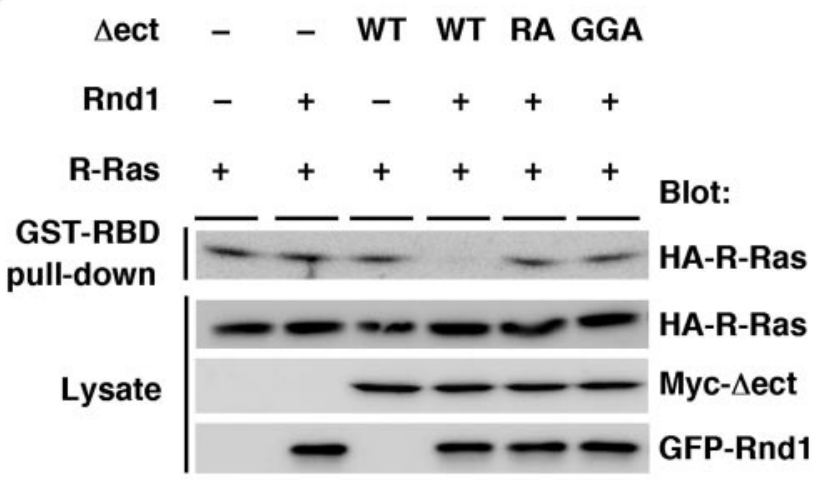

B

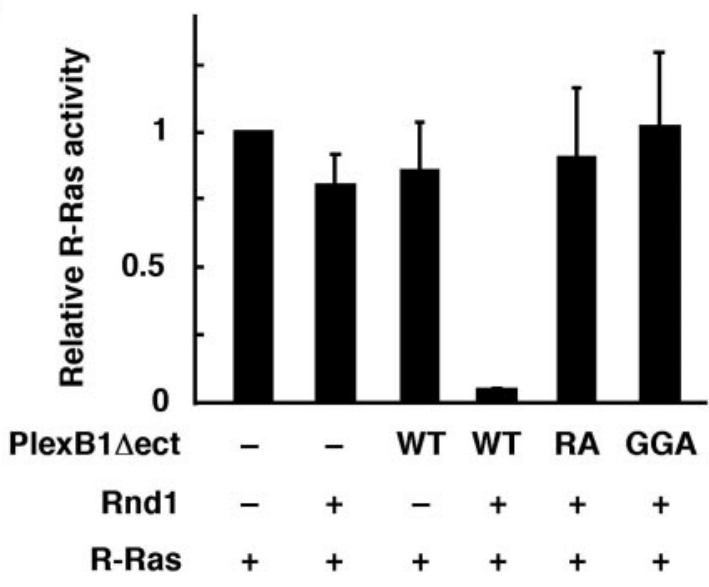

Figure 5. Rnd 1 binding is required for the GAP activity mediated by Plexin-B1 $\triangle$ ect. $A, C O S-7$ cells were transfected with Myc-tagged Plexin-B1 $\Delta$ ect mutants, GFP-Rnd1, and HA-R-Ras. GTP-bound R-Ras isolated with GST-RBD was detected with anti-HA antibody. B, Relative R-Ras activity was determined by the amount of R-Ras in cell lysates analyzed by NIH Image software. Results are the means \pm SEM of three independent experiments. WT, Wild type.

cytoplasmic domain of Plexin-B1, suggesting that this $\mathrm{C} 1-\mathrm{C} 2$ interaction renders the receptor inactive for R-Ras GAP activity. Indeed, the recombinant cytoplasmic domain alone does not show R-Ras GAP activity. Rnd 1 binding to the region between $\mathrm{C} 1$ and $\mathrm{C} 2$ domains disrupts the interaction of the $\mathrm{N}$-terminal region with the $\mathrm{C}$-terminal region, indicating that Rnd1 relieves the closed conformation of the cytoplasmic domain of Plexin-B1. We recently showed that interaction of the recombinant cytoplasmic domain of Plexin-B1 with the GTP-bound active form of R-Ras requires Rnd1 binding (Oinuma et al., 2004). Thus, the closed conformation cannot associate with GTP-bound R-Ras, whereas the Rnd1-bound open conformation acquires an ability to associate with GTP-bound R-Ras; that is, Rnd1 allows the receptor accessible for GTP-bound R-Ras. However, the Rnd1-bound recombinant Plexin-B1 cytoplasmic domain shows no R-Ras GAP activity and apparently suppresses the basal GTPase activity of R-Ras, suggesting that the Rnd1-bound receptor binds to GTPbound R-Ras and holds the G-protein as the GTP-bound active state. For the stimulation of GTP hydrolysis of R-Ras, clustering of the receptor is required as a second step.

Here we examined a role of Sema4D in the expression of R-Ras GAP activity of Plexin-B1. Sema4D induces clustering of Plexin-B1, in parallel with inactivation of R-Ras activity in cells. Direct clustering of the Rnd1-bound Plexin-B1 cytoplasmic domain by an antibody triggers R-Ras GAP activation, indicating that the receptor clustering is required and sufficient for the ex-
A
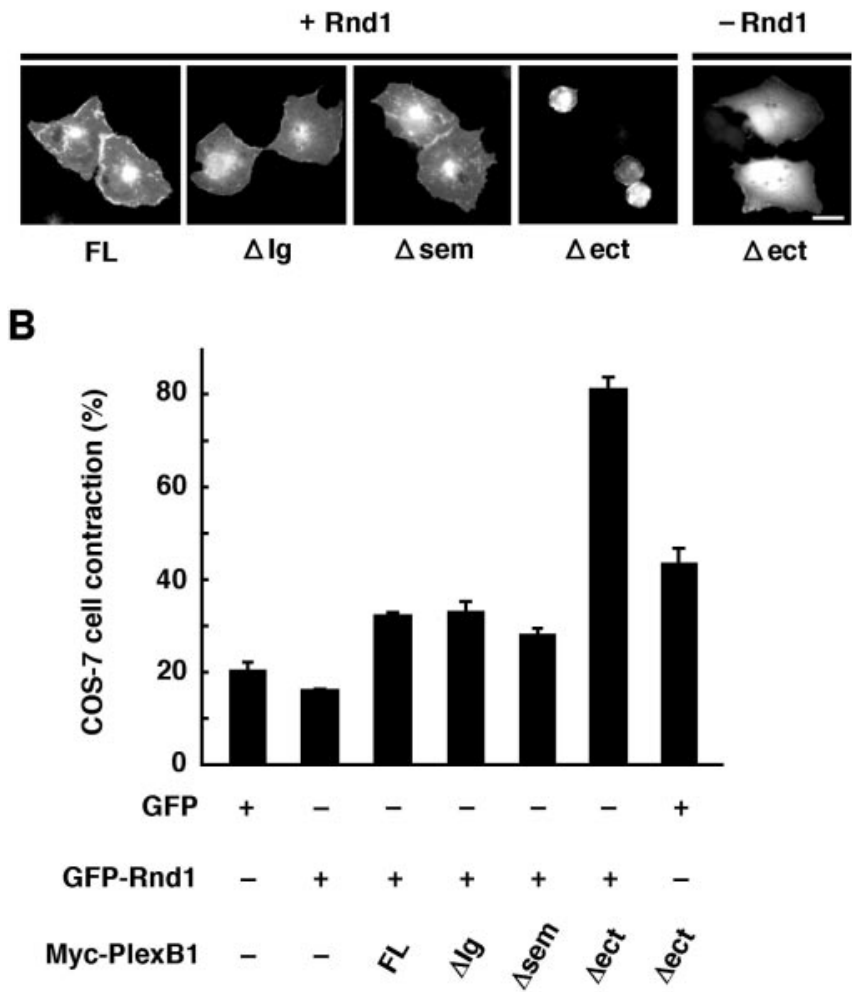

Figure 6. Plexin-B1 $\Delta$ ect induces ligand-independent contraction of $\operatorname{COS}-7$ cells. $A, \operatorname{COS}-7$ cells transfected with various deletion mutants of Plexin-B1 together with GFP or GFP-Rnd1 were fixed $14 \mathrm{hr}$ after transfection. Expression of Myc-tagged Plexin-B1 constructs was also detected with anti-Myc antibody (data not shown). Scale bar, $20 \mu \mathrm{m}$. B, Quantitative analysis of COS-7 cell contraction induced by Rnd1 and the deletion mutants of Plexin-B1. GFP- and Myc-staining double-positive cells with an area of $<350 \mu \mathrm{m}^{2}$ were scored as a percentage of the total number of transfected cells. Approximately 50 cells were assessed in one experiment, and data are the means \pm SEM of three independent experiments. FL, Full length.

pression of R-Ras GAP activity of Plexin-B1. Furthermore, we showed that the extracellular domain-deleted mutant of Plexin$\mathrm{B} 1$, Plexin-B1 $\Delta$ ect, forms a clustered receptor complex in the absence of Sema4D and displays constitutively active R-Ras GAP activity in the presence of Rnd1, leading to ligand-independent contraction of COS-7 cells and inhibition of neurite outgrowth in hippocampal neurons. These results strongly confirm that the clustering of Rnd1-bound Plexin-B1 stimulates R-Ras GAP activity and is enough for subsequent induction of morphological changes in COS-7 cells and neurons. A variety of tyrosine kinase receptors, such as epidermal growth factor and nerve growth factor receptors, are known to be activated via the clustering by their ligands (Sclessinger and Ullrich., 1992; Blakely et al., 2000; Burgess et al., 2003). On ligand binding, receptors undergo dimerization, and this physical clustering of the receptors causes trans-autophosphorylation of tyrosine residues within the receptors, leading to induction of their cellular functions. On the other hand, Plexin-B1 has the R-Ras GAP domain in the cytoplasmic region, which is separated by the Rnd1-binding domain into $\mathrm{C} 1$ and $\mathrm{C} 2$ domains. Rnd 1 binding relieves the closed conformation of the R-Ras GAP domain. The Rnd1-bound monomeric, open conformation of the domain can bind to GTP-bound R-Ras but has no ability to stimulate GTP hydrolysis. Clustering of the open conformation of the R-Ras GAP domain triggers the hydrolysis. This represents the fact that GAP activation of Plexin-B1 consists of two steps, interaction with GTP-bound R-Ras and promotion 
A
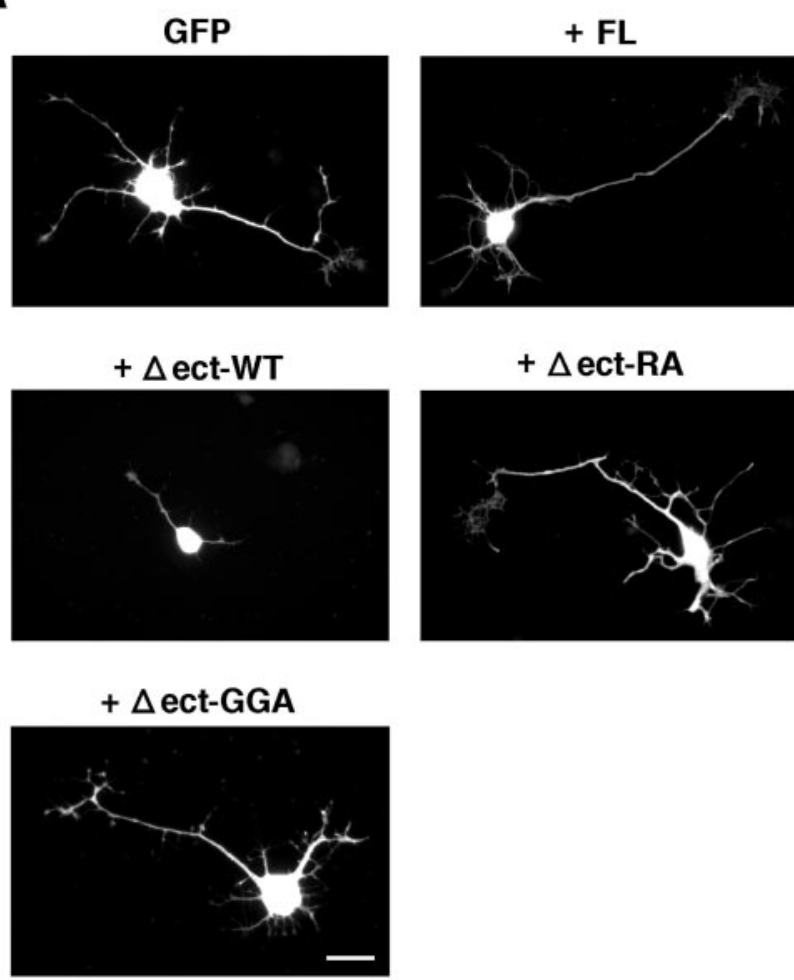

B

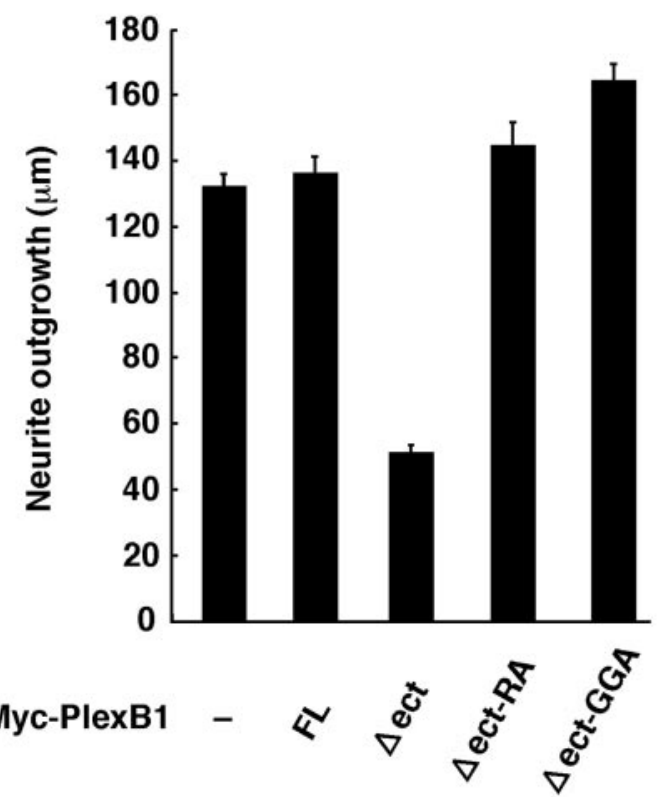

Figure 7. Plexin-B1 $\Delta$ ect is constitutively active in rat hippocampal neurons. $A$, Primary hippocampal neurons from rat embryos were seeded onto laminin-coated coverslips and cotransfected with GFP and Myc-tagged Plexin-B1. Cultures were fixed $16 \mathrm{hr}$ after transfection and stained with anti-Myc antibody. Transfected cells were shown by the fluorescence of GFP. Expression of Myc-tagged Plexin-B1 constructs was also detected with anti-Myc antibody (data not shown). Scale bar, $20 \mu \mathrm{m}$. B, Average neurite outgrowth was quantitated. The means \pm SEM of three independent experiments are presented. FL, Full length; WT, wild type.

of GTP hydrolysis by R-Ras; the former is getting ready by Rnd1, and the latter is a process induced by the clustering. A variety of GAPs for Ras family GTPases have been so far identified, and their GAP domains usually show high basal activity. The regula- tion of GAP activity is mediated by interaction with other molecules or receptors via various domains of GAPs, such as Src homology 2 (SH2) and SH3 (Bernards and Settleman, 2004). Considering the lack of basal R-Ras GAP activity of Plexin-B1, regulation of R-Ras GAP activity of Plexin-B1 by Rnd1 and clustering is a novel mechanism.

It has been reported that the sema domain of the Sema3A receptor, Plexin-A1, is an autoinhibitory domain, and deletion of the sema domain renders the receptor constitutively active (Takahashi and Strittmatter, 2001). In addition, Sema3A has been shown to induce the aggregation of Plexin-A on dorsal root ganglion growth cones (Takahashi et al., 1999). The R-Ras GAP homologous domains are well conserved among Plexin families, including Plexin-A1, and mutations in the GAP homologous domains in Plexin-A1 were reported to suppress the Plexin-A1-mediated repulsion (Rohm et al., 2000). Rnd1 binds to the cytoplasmic domain of Plexin-A1 and is required for the Sema3A- and Plexin-A1-mediated repulsion (Zanata et al. 2002). We recently reported that the downregulation of R-Ras GAP activity is also involved in the Sema3A-induced repulsion in hippocampal neurons (Oinuma et al., 2004). These results taken together suggest that Plexin-A exerts its function via an R-Ras GAP function regulated by Rnd 1 and receptor clustering similar to that of Plexin-B1.

Meanwhile, Plexin-B1 directly interacts with PDZ-RhoGEF/ LARG via its C-terminal PDZ domain-binding motif to induce RhoA activation and growth cone collapse in response to Sema4D (Swiercz et al., 2002). However, the RA mutant of Plexin-B1 $\Delta$ ect, which lacks the R-Ras GAP activity but has still the ability to interact with PDZ-RhoGEF, had no effect on the neurite outgrowth in hippocampal neurons. This result is consistent with previous reports obtained by using the ligandstimulated full length of Plexin-B1 (Barberis et al., 2004; Oinuma et al., 2004). These observations suggest that the Plexin-B1mediated morphological changes require both the R-Ras GAP activity and the PDZ-RhoGEF-mediated RhoA activation. We previously showed that Rnd1 binding to Plexin-B1 promotes the interaction between Plexin-B1 and PDZ-RhoGEF and thereby dramatically potentiates the Sema4D- and Plexin-B1-mediated RhoA activation (Oinuma et al., 2003). In this report, we revealed that Rnd1 binding to Plexin-B1 disrupts the interaction between the $\mathrm{N}$-terminal region and the $\mathrm{C}$-terminal region within the Plexin-B1 cytoplasmic domain. Considering that the RhoGEFbinding site is the C-terminal of Plexin-B1, this Rnd1-induced conformational change may promote the interaction of Plexin-B1 with PDZ-RhoGEF. Furthermore, Plexin-B1-PDZRhoGEF complex-mediated RhoA activation is triggered by Sema4D binding (Swiercz et al., 2002). Therefore, similar to R-Ras GAP activation, the Plexin-B1-mediated RhoA activation may also be critically regulated by Rnd1 binding and Sema4Dmediated receptor clustering.

$\mathrm{R}$-Ras promotes cell adhesion and controls cell migration and neurite outgrowth by activating integrins (Zhang et al., 1996; Ivins et al., 2000; Kinbara et al., 2003), and activation of Plexin-B1 inhibits integrin-mediated cell adhesion in a variety of cells (Barberis et al., 2004), suggesting that the downregulation of R-Ras activity by Plexin-B1 via the R-Ras GAP domain suppresses R-Ras-mediated integrin activation and thereby reduces cell adhesiveness, leading to growth cone collapse and inhibition of neurite outgrowth. Therefore, R-Ras GAP activity of Plexin-B1 is an important signaling system, and the regulation of R-Ras GAP activity is very critical for neuronal functions of Plexin-B1. 


\section{References}

Arevalo JC, Conde B, Hempstead BL, Chao MV, Martin-Zanca D, Perez P (2000) TrkA immunoglobulin-like ligand binding domains inhibit spontaneous activation of the receptor. Mol Cell Biol 20:5908-5916.

Aurandt J, Vikis HG, Gutkind JS, Ahn N, Guan KL (2002) The semaphorin receptor plexin-B1 signals through a direct interaction with the Rhospecific nucleotide exchange factor, LARG. Proc Natl Acad Sci USA 99:12085-12090.

Barberis D, Artigiani S, Casazza A, Corso S, Giordano C, Love CA, Jones EY, Comoglio PM, Tamagnone L (2004) Plexin signaling hampers integrinbased adhesion, leading to Rho-kinase independent cell rounding, and inhibiting lamellipodia extension and cell motility. FASEB J 18:592-594.

Bernards A, Settleman J (2004) GAP control: regulating the regulators of small GTPases. Trends Cell Biol 14:377-385.

Blakely BT, Rossi FM, Tillotson B, Palmer M, Estelles A, Blau HM (2000) Epidermal growth factor receptor dimerization monitored in live cells. Nat Biotechnol 18:218-222.

Burgess AW, Cho HS, Eigenbrot C, Ferguson KM, Garrett TP, Leahy DJ, Lemmon MA, Sliwkowski MX, Ward CW, Yokoyama S (2003) An open-and shut case? Recent insights into the activation of EGF/ErbB receptors. Mol Cell 12:541-552.

Cole LA, Subbanagounder G, Mukhopadhyay S, Berliner JA, Vora DK (2003) Oxidized phospholipid-induced endothelial cell/monocyte interaction is mediated by a cAMP-dependent R-Ras/PI3-kinase pathway. Arterioscler Thromb Vasc Biol 23:1384-1390.

Drissens MH, Hu H, Nobes CD, Self A, Jordens I, Goodman CS, Hall A (2001) Plexin-B semaphorin receptors interact directly with active Rac and regulate the actin cytoskeleton by activating Rho. Curr Biol 11:339-344.

Etienne-Manneville S, Hall A (2002) Rho GTPases in cell biology. Nature 420:629-635.

Hirotani M, Ohoka Y, Yamamoto T, Nirasawa H, Furuyama T, Kogo M, Matsuya T, Inagaki S (2002) Interaction of plexin-B1 with PDZ domain-containing Rho guanine nucleotide exchange factors. Biochem Biophys Res Commun 297:32-37.

Ishikawa Y, Katoh H, Negishi M (2003) A role of Rnd1 GTPase in dendritic spine formation in hippocampal neurons. J Neurosci 23:11065-11072.

Ivins JK, Yurchenco PD, Lander AD (2000) Regulation of neurite outgrowth by integrin activation. J Neurosci 20:6551-6560.

Jing S, Tapley P, Barbacid M (1992) Nerve growth factor mediates signal transduction through trk homodimer receptors. Neuron 9:1067-1079.

Katoh H, Harada A, Mori K, Negishi M (2002) Socius is a novel Rnd1GTPase interacting protein involved in disassembly of actin stress fibers. Mol Cell Biol 22:2952-2964.

Kinbara K, Goldfinger LE, Hansen M, Chou FL, Ginsberg MH (2003) Ras GTPases: integrins' friends or foes? Nat Rev Cell Biol 4:767-776.

Kolodkin AL, Matthes DJ, Goodman CS (1993) The semaphorin genes encode a family of transmembrane and secreted growth cone guidance molecules. Cell 75:1389-1399.

Luo Y, Raible D, Raper JA (1993) Collapsin: a protein in brain that induces the collapse and paralysis of neuronal growth cones. Cell 75:217-227.

Nakamura F, Kalb RG, Strittmatter SM (2000) Molecular basis of semaphorin-mediated axon guidance. J Neurobiol 44:219-229.
Negishi M, Katoh H (2002) Rho family GTPases as key regulators for neuronal network formation. J Biochem 132:157-166.

Nobes CD, Lauritzen I, Mattei MG, Paris S, Hall A, Chardin P (1998) A new member of the Rho family, Rnd1, promotes disassembly of actin filament structures and loss of cell adhesion. J Cell Biol 141:187-197.

Ohba Y, Mochizuki N, Yamashita S, Chan AM, Schrader JW, Hattori S, Nagashima K, Matsuda M (2000) Regulatory proteins of R-Ras, TC21/ R-Ras2, and M-Ras/R-Ras3. J Biol Chem 275:20020-20026.

Oinuma I, Katoh H, Harada A, Negishi M (2003) Direct interaction of Rnd1 with Plexin-B1 regulates PDZ-RhoGEF-mediated Rho activation by Plexin-B1 and induces cell contraction in COS-7 cells. J Biol Chem 278:25671-25677.

Oinuma I, Ishikawa Y, Katoh H, Negishi M (2004) The semaphorin 4D receptor Plexin-B1 is a GTPase activating protein for R-Ras. Science 305:862-865.

Perrot V, Vazquez-Prado J, Gutkind JS (2002) Plexin B regulates Rho through the guanine nucleotide exchange factors leukemia-associated Rho GEF (LARG) and PDZ-RhoGEF. J Biol Chem 277:43115-43120.

Raper JA (2000) Semaphorins and their receptors in vertebrates and invertebrates. Curr Opin Neurobiol 10:88-94.

Rohm B, Rahim B, Kleiber B, Hovatta I, Püschel AW (2000) The semaphorin $3 \mathrm{~A}$ receptor may directly regulate the activity of small GTPases. FEBS Lett 486:68-72.

Scheffzek K, Ahmandian M, Wittinghofer A (1998) GTP-ase activating proteins: helping hands to complement on active site. Trends Biochem Sci 23:257-262.

Sorokin A, Lemmon MA, Ulrich A, Schlessinger J (1994) Stabilization of an active dimeric form of the epidermal growth factor receptor by introduction of an inter-receptor disulfide bond. J Biol Chem 269:9752-9759.

Swiercz JM, Kuner R, Behrens J, Offermanns S (2002) Plexin-B1 directly interacts with PDZ-RhoGEF/LARG to regulate RhoA and growth cone morphology. Neuron 35:51-63.

Swiercz JM, Kuner R, Offermanns S (2004) Plexin-B1/RhoGEF-mediated RhoA activation involves the receptor tyrosine kinase ErbB-2. J Cell Biol 165:869-880.

Takahashi T, Strittmatter SM (2001) PlexinA1 autoinhibition by the plexin sema domain. Neuron 29:429-439.

Takahashi T, Fournier A, Nakamura F, Wang LH, Murakami Y, Kalb RG, Fujisawa H, Strittmatter SM (1999) Plexin-Neuropilin-1 complexes form functional Semaphorin-3A receptors. Cell 99:59-69.

Tamagnone L, Artigiani S, Chen H, He Z, Ming GI, Song H, Chedotal A, Winberg ML, Goodman CS, Poo M, Tessier-Lavigne M, Comoglio PM (1999) Plexins are large family of receptors for transmembrane, secreted, and GPI-anchored semaphorins in vertebrates. Cell 99:71-80.

van Triest M, de Rooij J, Bos JL (2001) Measurement of GTP-bound Ras-like GTPases by activation-specific probes. Methods Enzymol 333:343-348.

Vikis HG, Li W, He Z, Guan KL (2000) The semaphorin receptor plexin-B1 specifically interacts with active Rac in a ligand-dependent manner. Proc Natl Acad Sci USA 97:12457-12462.

Zanata SM, Hovatta I, Rohm B, Püschel AW (2002) Antagonistic effects of Rnd1 and RhoD GTPases regulate receptor activity in semaphorin 3Ainduced cytoskeletal collapse. J Neurosci 22:471-477.

Zhang Z, Vuori K, Wang H, Reed JC, Ruoslahti E (1996) Integrin activation by R-Ras. Cell 85:61-69. 